Y. Aihara and S. Mori

Nagoya Math. J.

Vol. 84 (1981), 209-218

\title{
ALGEBRAIC DEGENERACY THEOREM FOR HOLOMORPHIC MAPPINGS INTO SMOOTH PROJECTIVE ALGEBRAIC VARIETIES
}

\author{
YOSHIHIRO AIHARA AND SEIKI MORI
}

\section{§1. Introduction}

The famous Picard theorem states that a holomorphic mapping $f: C$ $\rightarrow \boldsymbol{P}^{1}(\boldsymbol{C})$ omitting distinct three points must be constant. Borel [1] showed that a non-degenerate holomorphic curve can miss at most $n+1$ hyperplanes in $\boldsymbol{P}^{n}(\boldsymbol{C})$ in general position, thus extending Picard's theorem $(n=1)$. Recently, Fujimoto [3], Green [4] and [5] obtained many Picard type theorems using Borel's methods for holomorphic mappings. In [3] and [4], they proved that a holomorphic mapping $f: \boldsymbol{C}^{m} \rightarrow \boldsymbol{P}^{n}(\boldsymbol{C})$ omitting any $n+2$ hyperplanes in general position must have the image lying in a hyperplane, especially Green showed that the same result holds under the condition that hyperplanes are distinct. Furthermore, in [5] he proved that a holomorphic mapping $f$ of $C^{m}$ into a projective algebraic variety $V$ of dimension $n$ omitting $n+2$ non-redundant hypersurface sections must be algebraically degenerate. On the other hand, in the equidimensional case, Carlson and Griffiths [2] obtained a generalization of Nevanlinna's defect relation for holomorphic mappings of $\boldsymbol{C}^{n}$ into an $n$-dimensional smooth projective algebraic variety $V$. By their results, a holomorphic mapping $f: C^{n} \rightarrow$ $\boldsymbol{P}^{n}(C)$ having the Nevanlinna's deficiency $\delta(D)=1$ for a hypersurface $D \subset$ $\boldsymbol{P}^{n}(C)$ of degree $\geqq n+2$ with simple normal crossings, must be degenerate in the sence that $J_{f} \equiv 0$ on $C^{n}$. While, Noguchi [6] obtained an inequality of the second main theorem type for holomorphic curves in algebraic varieties, thus a holomorphic curve $f$ in an algebraic variety $V$ which has the Nevanlinna's deficiency $\delta(\Sigma)=1$ for hypersurfaces $\Sigma$ with some conditions in $V$ must be algebraically degenerate. In this paper, we shall show that for $n+2$ ample divisors $\left\{D_{j}\right\}_{j=1}^{n+2}$ with normal crossings, any holomorphic mapping of $\boldsymbol{C}^{m}$ into an $n$-dimensional smooth projective algebraic variety

Received March 18, 1980. 
which has $\delta\left(D_{j}\right)=1(j=1, \cdots, n+2)$ must be algebraically degenerate. Hence a holomorphic mapping of $C^{n}$ into $P^{m}(C)$ with $\delta\left(H_{j}\right)=1(j=1, \cdots$, $n+2)$ for hyperplanes $\left\{H_{j}\right\}_{j=1}^{n+2}$ in $P^{n}(C)$ in general position must be linearly degenerate. Our method is different from that of Fujimoto and Green.

The authors express their thanks to Professor T. Katsura for his helpful conversations.

\section{§2. Notation and terminology}

Let $z=\left(z_{1}, \cdots, z_{m}\right)$ be the natural coordinate system in $C^{m}$. We set $\|z\|^{2}=\sum_{j=1}^{m} z_{j} \bar{z}_{j}, \quad B(r)=\left\{z \in C^{m} \mid\|z\|<r\right\}, \quad \partial B(r)=\left\{z \in C^{m} \mid\|z\|=r\right\}, \quad d^{c}=$ $(\sqrt{-1} / 4 \pi)(\bar{\partial}-\partial), \eta=d d^{c} \log \|z\|^{2}, \eta_{k}=\eta \wedge \cdots \wedge \eta(k$-times $)$ and $\sigma=d^{c} \log \|z\|^{2}$ $\wedge \eta_{m-1}$.

For a divisor $D(\supsetneqq 0)$ in $C^{m}$, we write

$$
n(D, t) \equiv \int_{D \cap B(t)} \eta_{m-1} \text { and } N(D, r) \equiv \int_{0}^{r} n(D, t)(d t / t)
$$

Let $V$ be an $n$-dimensional smooth projective algebraic variety and $L$ a line bundle over $V$. Let $\left\{U_{\alpha}\right\}$ be an open covering of $V$ such that the restriction $\left.L\right|_{U_{a}}$ is trivial. Then $L$ is determined by the 1-cocycle $\left\{f_{\alpha \beta}\right\}$ which are nowhere vanishing holomorphic functions in $U_{\alpha} \cap U_{\beta}$ satisfying $f_{\alpha \beta}=f_{\alpha \gamma} \cdot f_{\gamma \beta}$ in $U_{\alpha} \cap U_{\beta} \cap U_{r}$. A metric $h$ in $L$ is given by positive $C^{\infty}$ functions $h_{\alpha}$ in $U_{\alpha}$, where $h_{\alpha}=\left|f_{\alpha \beta}\right|^{2} h_{\beta}$ in $U_{\alpha} \cap U_{\beta}$. The curvature form $\omega$ of $h$ is given by $\omega=\omega_{L}=d d^{c} \log h_{\alpha}$ which represents the first Chern class $c_{1}(L)$ of $L$. A holomorphic line bundle $L$ on $V$ is said to be positive, if $L$ has a metric $h$ whose curvature form is everywhere positive definite.

Let $f$ be a holomorphic mapping of $C^{m}$ into $V$. Let $L$ be a positive line bundle over $V$ and $h$ a metric in $L$. We define

$$
T_{f}(L, r) \equiv \int_{0}^{r}(d t / t) \int_{B(t)} f^{*} \omega \wedge \eta_{m-1}
$$

and call it the characteristic function of $f$ with respect to $L$, where $f^{*} \omega$ denotes the pull-back of the form $\omega=d d^{c} \log h$ under $f$.

(*) We note that $T_{f}(L, r)$ is independent of the choice of a metric $h$ in $L$ up to $O(1)$-term. (See Carlson and Griffiths [2], p. 537).

A holomorphic section $\phi=\left\{\phi_{\alpha}\right\}$ of $L \rightarrow V$ is given by holomorphic functions $\phi_{\alpha}$ in $U_{\alpha}$ where $\phi_{\alpha}=f_{\alpha \beta} \phi_{\beta}$ in $U_{\alpha} \cap U_{\beta}$. For a section $\phi$, its norm $|\phi|$ is given by $|\phi|^{2}=\left|\phi_{\alpha}\right|^{2} / h_{\alpha}$ in $U_{\alpha}$ which is well defined on $V$. A holo- 
morphic line bundle whose sections defines a projective embedding is called very ample.

Let $\Gamma(V, \mathcal{O}(L))$ denote the space of holomorphic sections of the line bundle $L$ on $V$ and $|L|$ denote the complete linear system of effective divisors on $V$ given by the zeros of a holomorphic section of $L \rightarrow V$, i.e.

$$
|L|=\{(\phi) \mid \phi \in \Gamma(V, \mathcal{O}(L))\},
$$

where $(\phi)$ denotes the divisor given by the zeros of $\phi$.

Let $D \in|L|$ be an effective divisor given by the zeros of a holomorphic section $\phi \in \Gamma(V, \mathcal{O}(L))$ with $|\phi| \leqq 1$ on $V$. Assume that $\phi(f(z)) \not \equiv 0$. We define the proximity function of $D$ by

$$
m(D, r) \equiv \int_{\partial B(r)} \log \left(1 /|\phi|^{2}(f(z))\right) \sigma(z) \quad(\geqq 0) .
$$

Carlson and Griffiths [2] proved the following:

Theorem A (Carlson-Griffiths). Let $D \in|L|$ and $f: C^{m} \rightarrow V$ be a holomorphic mapping such that all components of $f^{*} D$ are divisors. Then

$$
N\left(f^{*} D, r\right)+m(D, r)=T_{f}(L, r)+O(1),
$$

where $O(1)$ depends on $D$ but not on $r$.

In the case where $f^{*} D$ passes through the origin, the definition of $N\left(f^{*} D, r\right)$ must be modified by means of Lelong numbers.

In the case that $V$ is an $n$-dimensional complex projective space $\boldsymbol{P}^{n}(\boldsymbol{C})$, Stoll [7] and Vitter [8] proved the Nevanlinna's second main theorem for meromorphic mappings of $\boldsymbol{C}^{m}$ into $\boldsymbol{P}^{n}(\boldsymbol{C})$ in the following form.

Theorem B (Stoll, Vitter). Let $f: \boldsymbol{C}^{m} \rightarrow \boldsymbol{P}^{n}(\boldsymbol{C})$ be a meromorphic mapping such that $f\left(\boldsymbol{C}^{m}\right)$ is not contained in any hyperplane in $\boldsymbol{P}^{n}(\boldsymbol{C})$. Let $\boldsymbol{H}$ be the hyperplane bundle over $\boldsymbol{P}^{n}(C)$ and $H_{1}, \cdots, H_{q} \in|H|$ distinct hyperplanes in general position in $\boldsymbol{P}^{n}(C)$. Then

$$
(q-n-1) T_{f}(H, r) \leqq \sum_{j=1}^{q} N\left(f^{*} H_{j}, r\right)+S(r),
$$

where $S(r) \leqq O\left(\log \left(r \cdot T_{f}(H, r)\right)\right)$ for $r \rightarrow \infty$ outside a set of finite Lebesgue measure.

For a divisor $D \in|L|$ on $V$, we define the deficiency of $D$ by

$$
\delta(D, r) \equiv 1-\limsup _{r \rightarrow \infty}\left(N\left(f^{*} D, r\right) / T_{f}(L, r)\right)
$$


Let $f$ be a holomorphic mapping of $C^{m}$ into a smooth projective algebraic variety $V$ such that $f\left(C^{m}\right)$ is not contained in any divisor belonging to $|L|$. Let $D_{1}, \cdots, D_{\ell}\left(D_{j} \in|L|\right)$ be divisors on $V$ given by the zeros of holomorphic sections $\phi_{1}, \cdots, \phi_{\ell}, \phi_{j}=\left\{\phi_{j_{\alpha}}\right\} \in \Gamma(V, \mathcal{O}(L))$ with $\left|\phi_{j}\right| \leqq 1(j=$ $1, \cdots, \ell)$ and the system $\left(\phi_{1}, \cdots, \phi_{\ell}\right)$ has no common zeros on $V$. Then the function $h=\left\{h_{\alpha}\right\}, h_{\alpha} \equiv \sum_{j=1}^{\ell}\left|\phi_{j \alpha}\right|^{2}$ is a positive $C^{\infty}$ function on $V$ and satisfies $h_{\alpha}=\left|f_{\alpha \beta}\right|^{2} h_{\beta}$ in $U_{\alpha} \cap U_{\beta}$. Hence we may take $h$ as a metric in $L$.

Note that, if $\psi_{1}$ and $\psi_{2}$ are two holomorphic sections of $L \rightarrow V$, then its ratio $\psi_{1} / \psi_{2}$ is a global meromorphic function on $V$.

By Theorem A, we have

$$
\begin{aligned}
T_{f}(L, r) & =N\left(f^{*} D_{i}, r\right)+m\left(D_{i}, r\right)+O(1) \\
& =N\left(f^{*} D_{i}, r\right)+\int_{\partial B(r)} \log \left(h_{\alpha}(f(z)) /\left|\phi_{i \alpha}\right|^{2}(f(z))\right) \sigma(z)+O(1) \\
& =N\left(f^{*} D_{i}, r\right)+\int_{\partial B(r)} \log \left(\sum_{j=1}^{b}\left|\phi_{j \alpha}(f(z)) / \phi_{i \alpha}(f(z))\right|^{2}\right) \sigma(z)+O(1) .
\end{aligned}
$$

\section{§3. Statement of results}

Let $V$ be a smooth projective algebraic variety of dimension $n$ and $L \rightarrow V$ a fixed positive line bundle over $V$. We shall prove the following theorem which yields an algebraic degeneracy of holomorphic mappings into $V$ under some conditions on the Nevanlinna's deficiencies.

TheOREM. Let $f: C^{m} \rightarrow V$ be a holomorphic mapping of $C^{m}$ into $V$. Let $D_{1}, \cdots, D_{n+2}, D_{j} \in\left|L^{\ell_{j}}\right|,\left(l_{j} \in Z^{+}\right)$, be divisors on $V$ such that $\delta\left(D_{j}\right)=1$ $(j=1, \cdots, n+2)$ and

$$
\bigcap_{k=1}^{n+1} \operatorname{supp} D_{j_{k}}=\emptyset \text { for every }\left\{j_{1}, \cdots, j_{n+1}\right\} \subset\{1, \cdots, n+2\} .
$$

Then $f$ must be algebraically degenerate.

Here $\delta\left(D_{j}\right)=1-\lim \sup _{r \rightarrow \infty}\left(N\left(f^{*} D_{j}, r\right) / T_{f}\left(L^{\ell_{j}}, r\right)\right)$ for $D_{j} \in\left|L^{\ell_{j}}\right|$ and $Z^{+}$ denotes the set of all positive integers.

We note that the condition (2) is satisfied for divisors $\left\{D_{j}\right\}_{j=1}^{n+2}$ with normal crossings.

Corollary. Let $S_{1}, \cdots, S_{n+2}$ be hypersurfaces with $\bigcap_{k=1}^{n+1} S_{j_{k}}=\emptyset$ in $\boldsymbol{P}^{n}(\boldsymbol{C})$ for every $\left\{j_{1}, \cdots, j_{n+1}\right\} \subset\{1, \cdots, n+2\}$. Then any holomorphic mapping $f: \boldsymbol{C}^{m} \rightarrow \boldsymbol{P}^{n}(\boldsymbol{C})$ which has $\delta\left(S_{j}\right)=1(j=1, \cdots, n+2)$ is algebraically degenerate. 
Remark. In this theorem, the condition (2) can not be replaced by a condition that $D_{1}, \cdots, D_{n+2}$ are non-redundant, i.e.

$$
\operatorname{supp} D_{j} \not \subset \bigcup_{i \neq j} \operatorname{supp} D_{i} \quad \text { for any } j \text {. }
$$

ExAmple. We consider a holomorphic curve $f: \boldsymbol{C} \rightarrow \boldsymbol{P}^{2}(\boldsymbol{C})$ given by $f=\left(1, e^{z}, z e^{z}\right)$ and four hyperplanes $H_{j}=\left\{w=\left(w_{1}, w_{2}, w_{3}\right) \in \boldsymbol{P}^{2}(\boldsymbol{C}) \mid w_{j}=0\right\}$ $(j=1,2,3)$ and $H_{4}=\left\{w \in \boldsymbol{P}^{2}(\boldsymbol{C}) \mid w_{3}-w_{2}=0\right\}$. Then we see that $N\left(f^{*} H_{j}, r\right)$ $=0$ for $j=1,2$ and $N\left(f^{*} H_{j}, r\right)=o\left(T_{f}(H, r)\right)$ for $j=3,4$ and hence $\delta\left(H_{j}\right)$ $=1$ for $j=1$ to 4 . But $f$ is not algebraically degenerate.

Remark. We can construct an example of a non-constant holomorphic curve in $P^{2}(C)$ which satisfies the conditions of the theorem for not all hyperplanes in $P^{2}(C)$.

\section{§4. Two lemmas}

In order to prove the theorem, we shall use the following two lemmas:

Lemma 1. Let $L \rightarrow V$ be a very ample line bundle over $V$ and $\psi_{1}, \cdots$, $\psi_{n+1}, \psi_{j}=\left\{\psi_{j \alpha}\right\} \in \Gamma(V, \mathcal{O}(L))$ holomorphic sections satisfying

$$
\bigcap_{j=1}^{n+1} \operatorname{supp} D_{j}=\emptyset
$$

where $D_{j}=\left(\psi_{j}\right)(j=1, \cdots, n+1)$. Then $\psi_{1}, \cdots, \psi_{n+1}$ are algebraically independent over $\boldsymbol{C}$.

Lemma 2. Let $\psi_{1}, \cdots, \psi_{n+2}, \psi_{j} \in \Gamma(V, \mathcal{O}(L))$ be holomorphic sections of a very ample line bundle $L \rightarrow V$ such that

$$
\bigcap_{k=1}^{n+1} \operatorname{supp} D_{j_{k}}=\emptyset \text { for every }\left\{j_{1}, \cdots, j_{n+1}\right\} \subset\{1, \cdots, n+2\},
$$

where $D_{j_{k}}=\left(\psi_{j_{k}}\right)(k=1, \cdots, n+1)$. Let $R\left(\psi_{1}, \cdots, \psi_{n+2}\right) \equiv \sum_{j=1}^{s} R_{j} \equiv 0$ be an algebraic relation of an irreducible homogeneous polynomial of degree $k$ in $\psi$ 's among $\psi_{1}, \cdots, \psi_{n+2}$. Then

$$
\left\{p \in V \mid R_{j_{1}}(p)=\cdots=R_{s_{s-1}}(p)=0\right\}=\emptyset
$$

for every $\left\{j_{1}, \cdots, j_{s-1}\right\} \subset\{1, \cdots, s\}$.

Proof of Lemma 1. Let $\zeta_{0}, \cdots, \zeta_{N}$ be a basis of global holomorphic sections of $L$. Since $L$ is very ample, the mapping $\Phi_{L}=\left(\zeta_{0}, \cdots, \zeta_{N}\right)$ gives a projective embedding of $V$ into $\boldsymbol{P}^{N}(C)$. We identify $V$ with $\Phi_{L}(V)$. By 
means of this embedding, we can identify $L$ with the restriction of the hyperplane bundle $H$ over $P^{N}(C)$ to $V$. Hence for each $\psi_{j} \in \Gamma(V, \mathcal{O}(L))$ there exist global holomorphic sections $\tilde{\psi}_{j} \in \Gamma\left(\boldsymbol{P}^{N}(C), \mathcal{O}(H)\right)$ such that $\left.\tilde{\psi}_{j}\right|_{v}=\psi_{j}$.

We set $\left(\tilde{\psi}_{j}\right)=\tilde{D}_{j}(j=1, \cdots, n+1)$. Hence the dimension of the algebraic subvarieties

$$
V_{j k} \equiv \operatorname{supp} \tilde{D}_{j} \cap \operatorname{supp} \tilde{D}_{k} \cap V
$$

in $V$ is not less than $(n-1)+(N-1)-N=n-2$, that is, $\operatorname{dim} V_{j k} \geqq$ $n-2$. Similarly, we see that the dimension of

$$
V_{j k \ell} \equiv V_{j k} \cap \operatorname{supp} \tilde{D}_{\ell} \cap V
$$

is not less than $n-3$. Repeating the same argument as above, we have

$$
\operatorname{dim}\left(\operatorname{supp} D_{j_{1}} \cap \cdots \cap \operatorname{supp} D_{j_{n}}\right) \geqq 0,
$$

that is,

$$
\operatorname{supp} D_{j_{1}} \cap \cdots \cap \operatorname{supp} D_{j_{n}} \neq \emptyset \text {. }
$$

Suppose that $\psi_{1}, \cdots, \psi_{n+1}$ have an algebraic relation $R$ of homogeneous polynomial of degree $k$ in $\psi_{1}, \cdots, \psi_{n+1}$ represented by

$$
R\left(\psi_{1}, \cdots, \psi_{n+1}\right) \equiv \sum_{i_{1}+\cdots+i_{n+1}=k} c_{i_{1} \cdots i_{n+1}} \psi_{1}^{i_{1}} \cdots \psi_{n+1}^{i_{n+1}} \equiv 0
$$

Then we see that $c_{0 . \ldots 0 k}=0$, since $\psi_{n+1}(p) \neq 0$ for a point $p \in V$ with $\psi_{1}(p)$ $=\cdots=\psi_{n}(p)=0$. Thus the term $\psi_{n+1}^{k}$ is not contained in the relation $R$. Similarly, we find that none of the terms $\psi_{1}^{k}, \cdots, \psi_{n+1}^{k}$ belongs to $R$.

We next consider the curve $\mathscr{L}=\left\{p \in V \mid \psi_{1}(p)=\cdots=\psi_{n-1}(p)=0\right\}$. For any point $p \in \mathscr{L}$, we see

$$
\sum_{i_{n}+i_{n+1}=k} c_{0 \ldots 0 i_{n} i_{n+1}} \psi_{n}^{i_{n}} \cdot \psi_{n+1}^{i_{n+1}} \equiv 0 \quad \text { on } \mathscr{L}
$$

We may assume that all $c_{0 \ldots i_{n} i_{n+1}}$ are not zero. Then we can rewrite (4) in the form

$$
\psi_{n}^{r_{n}} \cdot \psi_{n+1}^{r_{n+1}}\left\{\psi_{n+1}^{k_{n, n+1}}+c_{0 \ldots 0_{* *} *} \psi_{n+1}^{k_{n, n+1}-1} \cdot \psi_{n}+\cdots+c_{0 \ldots 0 * *}^{\prime} \psi_{n}^{k_{n, n+1}}\right\} \equiv 0
$$

on $\mathscr{L}$, where $r_{k}=\min i_{k}(k=n, n+1)$ and $k_{n, n+1}=k-\left(r_{n}+r_{n+1}\right),(\neq 0)$. Since $\psi_{n} \cdot \psi_{n+1} \not \equiv 0$ on $\mathscr{L}$, we obtain

$$
\psi_{n+1}^{k_{n, n+1}}+\cdots+c_{0 . \ldots 0^{*}}^{\prime} \psi_{n}^{k_{n, n+1}} \equiv 0 \quad \text { on } \mathscr{L}-\left\{\left(\psi_{n}=0\right)^{\cup}\left(\psi_{n+1}=0\right)\right\} \text {. }
$$

By Riemann's extension theorem, 


$$
\psi_{n+1}^{k_{n, n+1}}+\cdots+c_{0 . \ldots * *+1}^{\prime} \psi_{n}^{k_{n, n+1}} \equiv 0 \quad \text { on } \mathscr{L} .
$$

We now take a point $p_{n} \in \mathscr{L}$ with $\psi_{n}\left(p_{n}\right)=0$. Then we see $\psi_{n+1}\left(p_{n}\right)=0$ by (5). This is a contradiction. Thus any $c_{0 \ldots 0 i_{i n+1}}$ equals to zero, that is, no terms $\psi_{n}^{i_{n}} \cdot \psi_{n+1}^{i_{n+1}}$ are contained in $R$. Similarly, we see that no terms $\psi_{k}^{i_{k}} \cdot \psi_{\ell}^{i_{\ell}}$ are involved in $R$ for any $i_{k}, i_{\ell}$. We next consider the subvarieties

$$
\begin{aligned}
S(j, k, \ell) & =\left\{p \in V \mid \psi_{1}(p)=\cdots=\hat{\psi}_{\jmath}(p)\right. \\
& \left.=\cdots=\hat{\psi}_{k}(p)=\cdots=\hat{\psi}_{\ell}(p)=\cdots=\psi_{n+1}(p)=0\right\}
\end{aligned}
$$

and

$$
\begin{aligned}
L(j, k) & =\left\{p \in V \mid \psi_{1}(p)=\cdots=\hat{\psi}_{j}(p)\right. \\
& \left.=\cdots=\hat{\psi}_{k}(p)=\cdots=\psi_{n+1}(p)=0\right\},
\end{aligned}
$$

where the $\wedge$ over the $\psi_{j}$ means that this terms is to be omitted. Then the similar argument to the above implies that no terms of products of three $\psi$ 's are involved in $R$. Repeating the above argument, we have the fact that all coefficients $c_{i_{1} \cdots i_{n+1}}$ in $R$ are equal to zero, that is, $\psi_{1}, \cdots, \psi_{n+1}$ are algebraically independent. This completes the proof of Lemma 1.

Proof of Lemma 2. From the condition (3), the mapping $\Psi: V \rightarrow P^{n+1}(C)$ given by $V \ni p \mapsto\left(\psi_{1}(p), \cdots, \psi_{n+2}(p)\right) \in \boldsymbol{P}^{n+1}(C)$ is well defined and holomorphic. By Remmert's proper mapping theorem, $\Psi(V)$ is an analytic subset of $\boldsymbol{P}^{n+1}(\boldsymbol{C})$, hence it is algebraic in $\boldsymbol{P}^{n+1}(\boldsymbol{C})$. We note that any $n+1$ $\psi$ 's in $\psi_{1}, \cdots, \psi_{n+2}$ are algebraically independent by Lemma 1 . Then using elimination theory, we see that $\Psi(V)$ is an irreducible hypersurface $R$ in $\boldsymbol{P}^{n+1}(C)$. We write the $R$ in $\boldsymbol{P}^{n+1}(C)$ as

$$
R\left(x_{1}, \cdots, x_{n+2}\right) \equiv \sum_{i_{1}+\cdots+i_{n+2}=k} a_{i_{1} \cdots i_{n+2}} x_{1}^{i_{1}} \cdots x_{n+2}^{i_{n}} \equiv 0
$$

for a homogeneous coordinate system $\left(x_{1}, \cdots, x_{n+2}\right)$ in $P^{n+1}(C)$.

We now consider the point $(1,0, \cdots, 0) \in \boldsymbol{P}^{n+1}(\boldsymbol{C})$. Then we see $(1,0, \cdots, 0) \notin R$ from the hypothesis (3) in $\psi_{1}, \cdots, \psi_{n+2}$.

Thus we see $a_{k 0 \ldots 0} \neq 0$. Similarly, we have

$$
a_{0 k \ldots 0} \neq 0, \cdots, a_{0 \ldots 0 k} \neq 0 \text {. }
$$

Thus we can rewrite (6) in the form

$$
R\left(x_{1}, \cdots, x_{n+2}\right)=a_{k 0 \ldots 0} x_{1}^{k}+\cdots+a_{0 \ldots 0 k} x_{n+2}^{k}+\alpha\left(x_{1}, \cdots, x_{n+2}\right),
$$

where $\alpha\left(x_{1}, \cdots, x_{n+2}\right)$ are the remainder terms of $R$. Hence we obtain 


$$
\begin{aligned}
R\left(\psi_{1}, \cdots, \psi_{n+2}\right) & =a_{k 0 \cdots 0} \psi_{1}^{k}+\cdots+a_{0 \cdots 0 k} \psi_{n+2}^{k}+\alpha\left(\psi_{1}, \cdots, \psi_{n+2}\right) \\
& \equiv R_{1}+\cdots+R_{n+2}+R_{n+3}+\cdots+R_{s}, \quad \text { (say), }
\end{aligned}
$$

where $R_{j}=a_{0 \ldots 0 k \ldots, \ldots 0}^{(j)} \psi_{j}^{k}$ and $a_{0 \ldots 0 k 0 \ldots 0}^{(j)} \neq 0(j=1, \cdots, n+2)$. Therefore we see $\left\{p \in V \mid R_{f_{1}}(p)=\cdots=R_{f_{s-1}}(p)=0\right\}=\emptyset$ for every $\left\{j_{1}, \cdots, j_{s-1}\right\} \subset\{1, \cdots, s\}$ by means of $\left\{p \in V \mid R_{i_{1}}(p)=\cdots=R_{i_{n+1}}(p)=0\right\}=\emptyset$ for every $\left\{i_{1}, \cdots, i_{n+1}\right\}$ $\subset\{1, \cdots, n+2\}$ and $s \geqq n+2$. This completes the proof of Lemma 2 .

\section{§5. Proof of Theorem}

By the definition of divisors $\left\{D_{j}\right\}$, there exist holomorphic sections $\tilde{\phi}_{j}$ $\in \Gamma\left(V, \mathcal{O}\left(L^{\ell_{j}}\right)\right)$ such that $D_{j}=\left(\tilde{\phi}_{j}\right)$ and $\left|\tilde{\phi}_{j}\right| \leqq 1$ for $j=1, \cdots, n+2$. Let $\ell_{0}=$ l.c.m. $\left(\ell_{1}, \cdots, \ell_{n+2}\right)$ and $\ell=N \ell_{0}$ for some $N \in Z^{+}$so that the line bundle $L^{\ell}$ becomes very ample. We set $\phi_{j}=\tilde{\phi}_{j}^{\ell / \ell_{j}}$. Then $\phi_{j}$ belongs to $\Gamma\left(V, \mathcal{O}\left(L^{\ell}\right)\right)$ $(j=1, \cdots, n+2)$, and $\left\{\phi_{j} / \phi_{i}\right\}$ are global meromorphic functions on $V$. Since $V$ has a transcendence degree $n$, there exists a relation $R$ of an irreducible homogeneous polynomial in $\phi_{1}, \cdots, \phi_{n+2}$. We write

$$
R\left(\phi_{1}, \cdots, \phi_{n+2}\right) \equiv \sum_{j=1}^{s} R_{j} \equiv 0 \text {. }
$$

Then for every $\left\{j_{1}, \cdots, j_{s-1}\right\} \subset\{1, \cdots, s\},\left(R_{j_{1}}, \cdots, R_{j_{s-1}}\right)$ has no common zero points by Lemma 2 (say, $\left\{R_{1}, \cdots, R_{s-1}\right\}$ ), since $L^{\ell}$ is a very ample line bundle over $V$ and supp $\left(\left(\phi_{j}\right)\right)=\operatorname{supp}\left(\left(\tilde{\phi}_{j}\right)\right)$. Furthermore, it is clear that $R_{j} \in \Gamma\left(V, \mathcal{O}\left(L^{d}\right)\right)$ for some $d \in Z^{+}$. We set $h=\sum_{j=1}^{s-1}\left|R_{j}\right|^{2}$. Then $h$ is a positive $C^{\infty}$ function with $h_{\alpha}=\left|f_{\alpha \beta}\right|^{2} h_{\beta}$, where $L^{d}=\left\{f_{\alpha \beta}\right\}$. Thus $h$ is a metric in the line bundle $L^{d} \rightarrow V$. We note that from (*) and the definition of $T_{f}(L, r)$,.

$$
T_{f}\left(L^{d}, r\right)=d \cdot T_{f}(L, r)+O(1)
$$

for any choice of a metric $h$ in $L^{d}$. From (1) and (8), we have

$$
T_{f}\left(L^{d}, r\right)=\int_{\partial B(r)} \log \left(f^{*} h /\left|f^{*} R_{\jmath}\right|^{2}\right) \sigma+N\left(f^{*}\left(R_{j}\right), r\right)+O(1)
$$

where $\left(R_{j}\right)$ denotes the divisor in $V$ given by the zeros of $R_{j}, f^{*}\left(R_{j}\right)$ denotes the pull back divisor of $\left(R_{j}\right)$ in $C^{m}$ and $f^{*} R_{j}$ is the pull back of the section $R_{\text {, }}$ under $f$.

Now we consider a holomorphic mapping from $C^{m}$ into $P^{s-2}(C)$ with the representation $F=\left(f^{*} R_{1}, \cdots, f^{*} R_{s-1}\right): C^{m} \rightarrow P^{s-2}(C)$. Let $H$ be the hyperplane bundle over $P^{s-2}(C)$. Taking the Fubini-Study metric in $H$, we see from Theorem A 


$$
T_{F}(H, r)=\int_{\partial B(r)} \log \left(\left.\sum_{j=1}^{s-1}\left|f^{*} R_{\jmath}\right| f^{*} R_{i}\right|^{2}\right) \sigma+N\left(f^{*}\left(R_{i}\right), r\right)+O(1)
$$

Hence from (9) and (10), we have

$$
T_{F}(H, r)=T_{f}\left(H^{d}, r\right)+O(1) .
$$

We now consider the following $s$ hyperplanes $H_{1}, \cdots, H_{s}$ in $P^{s-2}(C)$ in general position; for a homogeneous coordinate system $t=\left(t_{1}, \cdots, t_{s-1}\right)$ in $\boldsymbol{P}^{s-2}(\boldsymbol{C}), H_{j}=\left\{t \in \boldsymbol{P}^{s-2}(\boldsymbol{C}) \mid t_{j}=0\right\}(j=1, \cdots, s-1)$ and $H_{s}=\left\{t \in \boldsymbol{P}^{s-2}(\boldsymbol{C}) \mid \sum_{j=1}^{s-1} t_{j}\right.$ $=0\}$. The hypothesis $\delta\left(D_{i}\right)=1-\lim \sup _{r \rightarrow \infty} N\left(f^{*} D_{j}, r\right) / T_{f}\left(L^{\ell_{j}}, r\right)=1$ implies that

$$
N\left(F^{*} H_{j}, r\right)=O\left(\sum_{i=1}^{n+2} N\left(f^{*} D_{i}, r\right)\right)=o\left(\sum_{i=1}^{n+2} T_{f}\left(L^{\ell_{i}}, r\right)\right)=o\left(T_{F}(H, r)\right)
$$

for $j=1, \cdots, s-1$ and

$$
N\left(F^{*} H_{s}, r\right)=N\left(f^{*}\left(R_{s}\right), r\right)=o\left(T_{F}(H, r)\right) .
$$

Suppose first that $F$ is rational. Note that $F$ is rational if and only if $T_{F}(H, r)=O(\log r)$. Then $N\left(F^{*} H_{j}, r\right)=o\left(T_{F}(H, r)\right)$ implies that $F\left(C^{m}\right)$ $\cap H_{j}=\emptyset(j=1, \cdots, s)$. Thus $f^{*} R_{j} / f^{*} R_{i} \neq 0$ and is rational on $C^{m}$, and hence it is constant on $C^{m}$. Thus $f^{*} R_{j}-c f^{*} R_{i}=0$ for some constant $c$, that is, $f\left(C^{m}\right)$ lies in the hypersurfaces $R_{j}-c R_{i}=0$ in $V$ for $i, j=1, \cdots, s$.

Finally, we assume that $F$ is transcendental. Suppose that $F$ is not linearly degenerate. Using Theorem $\mathrm{B}$ with $s=q$ and $n=s-2$, we have

$$
T_{F}(H, r) \leqq o(T(H, r))+O\left(\log \left(r \cdot T_{F}(H, r)\right)\right)
$$

for $r \rightarrow \infty$ outside a set of finite Lebesgue measure. This is absurd. Thus $F$ is linearly degenerate, that is, there exist constants $\left(c_{1}, \cdots, c_{s-1}\right) \in C^{s-1}$ $-\{0\}$ such that

$$
c_{1} f^{*} R_{1}+\cdots+c_{s-1} f^{*} R_{s-1} \equiv 0 .
$$

Hence the image $f\left(C^{m}\right)$ lies in the hypersurface given by

$$
c_{1} R_{1}+\cdots+c_{s-1} R_{s-1} \equiv 0 \text {. }
$$

Therefore $f$ is algebraically degenerate. This completes the proof of the theorem.

Remark. The theorem holds for a meromorphic mapping of $C^{m}$ into a smooth projective algebraic variety $V$. 


\section{REFERENCES}

[1] E. Borel, Sur les zéros des fonctions entières, Acta Math., 20 (1896), 357-396.

[2] J. Carlson and P. A. Griffiths, A defect relation for equidimensional holomorphic mappings between algebraic varieties, Ann. of Math., 95 (3) (1972), 556-584.

[ 3 ] H. Fujimoto, Extensions of the big Picard's theorem, Tôhoku Math. J., 24 (1972), 415-422.

[4] M. L. Green, Holomorphic maps into complex projective space omitting hyperplanes, Trans. Amer. Math. Soc., 169 (1972), 80-103.

[5] - Some Picard theorems for holomorphic maps to algebraic varieties, Amer. J. Math., 97 (1975) , 43-75.

[6] J. Noguchi, Holomorphic curves in algebraic varieties, Hiroshima Math. J., 7 (1977), 833-853.

[7] W. Stoll, Die beiden Hauptsätze der Wertverteilungs theorie bei funktionen mehrerer komplexen Veränderlichen (I) (II), Acta Math., 90 (1953), 1-115, and 92 (1954), 55-169.

[8] A Vitter, The lemma of the logarithmic derivative in several complex variables, Duke Math. J., 44 (1977), 89-104.

Mathematical Institute

Tôhoku University

Sendai, 980 Japan 